\title{
Magnetic field-induced vortex triplet and vortex lattice in a liquid crystal cell
}

\author{
Enrique Calisto $\odot,{ }^{1,2}$ Marcel G. Clerc $\odot,{ }^{1}$ and Valeska Zambra $\odot^{1}$ \\ ${ }^{1}$ Departamento de Física and Millennium Institute for Research in Optics, FCFM, Universidad de Chile, Casilla 487-3, Santiago, Chile \\ ${ }^{2}$ Departamento de Ingeniería Matemática, FCFM, Universidad de Chile, Casilla 170-3. Correo 3, Santiago, Chile
}

(Received 4 June 2020; accepted 20 October 2020; published 10 November 2020)

\begin{abstract}
Vortices are particle-type solutions with topological charges that can steer the dynamics in various physical systems. By the application of electromagnetic fields onto a homeotropic nematic liquid crystal cell, we are able to induce a vortex triplet that remains stable and trapped at a given location. For a low frequency of the driven voltage, we observe that the vortex triplet is unstable and gives rise to the appearance of a topological lattice. Based on an amplitude equation valid close to reorientational instability, it allows us to reveal the origin of the vortex triplet and vortex lattice. Numerical simulations show a quite fair agreement with theoretical findings and experimental observations.
\end{abstract}

DOI: 10.1103/PhysRevResearch.2.042026

Continuous media are characterized by exhibiting different robust phenomena such as waves, fronts, patterns, and dissipative structures [1-4]. One of the most attractive dynamic behaviors is particle-type solutions or localized structures, where solitons are the paradigmatic example $[5,6]$. These dynamic behaviors are extended spatially; however, they exhibit characteristics associated with particles such as a position, amplitude, charge, width, among others. The localized structures are understood as patterns appearing in a restricted region of space [7-10]. Dissipative particle-type solutions have been theoretically predicted and experimentally observed in many fields of nonlinear science, such as laser physics, hydrodynamics, fluidized granular media, gas discharge systems, chemical reactions, magnetic media, and biology (see the reviews in Refs. [7-10] and references therein). Localized structures can be created or destroyed by localized disturbances [7-11]. All these features change radically when one considers particle-type solutions with topological charges, called vortices [12]. Vortices are pointlike singularities that take place in complex fields, which locally break the rotation symmetry. They present zero intensity at the singular point with a phase spiraling around it. The topological charge is allocated by counting the number of phase jumps, while the sign is given by the direction of the spiral rotation. Conventionally, it is positive (negative) when the rotation is counterclockwise (clockwise). As a consequence of the conservation of topological charge, vortices are created and annihilated by pairs between opposite charges [12]. In addition, this conservation precludes that a local disturbance would destroy vortices. Indeed, the possession of a topological charge provides vortices with a topological stability, as occurs with fundamental particles [12]. A paradigmatic field model that presents vortices is the Ginzburg-Landau equation $[12,13]$. This amplitude

Published by the American Physical Society under the terms of the Creative Commons Attribution 4.0 International license. Further distribution of this work must maintain attribution to the author(s) and the published article's title, journal citation, and DOI. equation has been used to describe fluids, superfluids, superconductors, liquid crystals, granular matter, magnetic media, and optical dielectrics, to mention a few [12-15].

A natural physical context where dissipative vortices are observed is in liquid crystals [16,17]. Vortices in this context are usually called umbilical defects or disclination lines. Considering a thin film of nematic liquid crystal with a negative anisotropic dielectric constant and homeotropic anchoring, applying a sufficiently large transverse electric field generates a molecular reorientation, known as the Fréedericksz transition [18]. This transition is characterized by the spontaneous emergence of a vortex gas. Due to the attraction and annihilation of vortex pairs, the system ultimately finds its homogeneous equilibrium state, characterized by the fact that the molecules in the bulk are misaligned from the applied electric field [16,17]. These topological defects are of great relevance because when interacting with light they can be a source of optical vortex beams [19-22]. Alternatively, one can induce umbilical defects by means of intense light beams [23], photosensitive walls [21,24], or by means of external magnetic and electric fields [25,26]. The combined action of a distant magnetic ring with a uniform electric field into the nematic cell enables the self-engineering of macroscopic $q$ plates [26]. The possibility of manipulating vortices allows creating optical vortex lattices, which are useful for quantum computation [27], image analysis [28], and generation and data transmission [29].

This Rapid Communication aims to show that a vortex triplet is induced by the combined action of a close magnetic ring and oscillatory electric field into a nematic liquid crystal layer. The vortex triplet is characterized by a positively charged vortex in the center and two vortices on its flanks of opposite charges. Namely, the triplet has a positive total topological charge consistent with that enforced by the magnetic ring. The vortex triplet is a consequence of the structure of the magnetic protuberance at the ring center, vertical electric field, and inherent anisotropy of the liquid crystal. For a low frequency of the driven voltage, we observe that the vortex triplet is unstable and gives rise to the appearance of a topological lattice. An amplitude equation allows us to explain the 
(a)

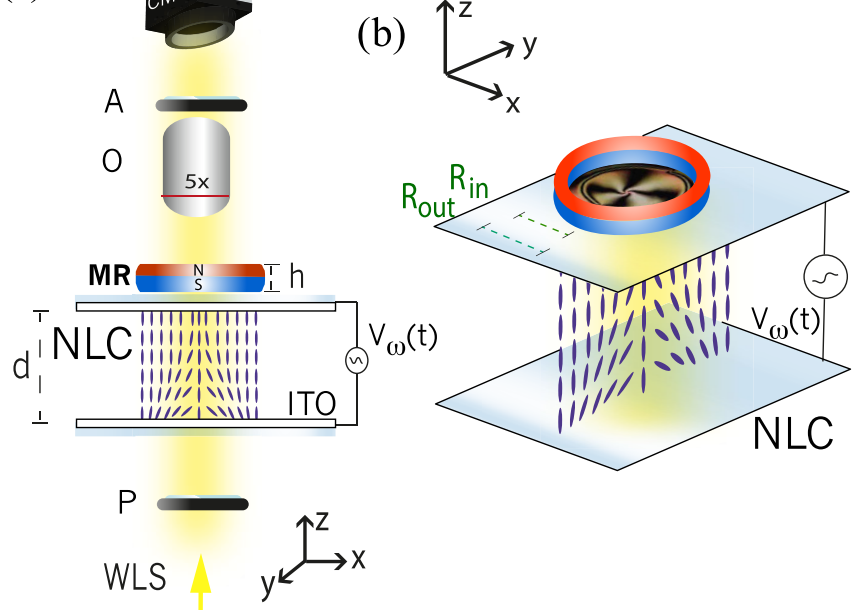

FIG. 1. Schematic representation of the experimental setup. (a) Vertical description of the experimental setup. NLC accounts for the nematic liquid crystal cell, $d$ is the thickness of the cell, ITO accounts for the transparent electrode (indium tin oxide), $V_{\omega}(t)$ is a harmonic voltage applied to the liquid crystal layer, where $\omega$ is the voltage frequency, MR is the magnetic ring of thickness $h$ and internal and outer radius $R_{\text {in }}$ and $R_{\text {out }}$, WLS is the white light source, $P$ and $A$ stand for polarizers, which are crossed, $O$ accounts for the objective, and CMOS is the complementary metal-oxide-semiconductor camera. The rods represent the average direction of molecules, directors. (b) Three-dimensional schematic representation of the liquid crystal cell under the influence of electric and magnetic fields.

emergence of a vortex triplet and vortex lattice. Numerical simulations show a good agreement with experimental observations.

Experimental setup. Figure 1 shows a schematic representation of the experimental setup. To study the vortex dynamics, we consider a cell composed of two thin glass layers separated by a thickness of $d=75 \mu \mathrm{m}$, which is chemically treated on its interior walls to have a homogeneous homeotropic anchoring and with transparent electrodes included [indium tin oxide (ITO) with a thickness of $0.08 \mu \mathrm{m}$ ]. This cell has been filled by capillarity with a nematic liquid crystal LC-BYVA-01-5G (Instec) with negative dielectric anisotropy $\epsilon_{a}=-4.89$, birefringence $\Delta n=n_{e}-n_{o}=0.1$, rotation viscosity $\gamma=204 \mathrm{mPa}$ s, splay and bend elastic constant, respectively, $K_{1}=17.65 \mathrm{pN}$ and $K_{3}=21.39 \mathrm{pN}$, and negative magnetic anisotropy $\chi_{a}$ (not yet measured). All experiments were conducted at a room temperature of $21^{\circ} \mathrm{C}$. A neodymium magnetic ring of $3200 \mathrm{G}$ with a rectangular transversal section, outer radius $R_{\text {out }}=7 \mathrm{~mm}$, internal radius $R_{\text {in }}=2 \mathrm{~mm}$, and thickness of $h=5 \mathrm{~mm}$ is put on top of the nematic liquid crystal cell (cf. Fig. 1). The sample with the magnetic ring is introduced in an Olympus $\mathrm{B} \times 51$ microscope and it is sandwiched between two linear cross polarizers. A sinusoidal voltage of intensity $V_{0}=7.95 \mathrm{~V}_{\mathrm{pp}}$, near the reorientational transition, and a Fréedericksz voltage $V_{\mathrm{FT}}=6.57$ $\mathrm{V}_{\mathrm{pp}}$, with a frequency of $\omega=100 \mathrm{~Hz}$ is applied to the sample. The system is illuminated by a white light (halogen lamp). The temporal evolution of the liquid crystal cell under the simultaneous effects of the electric and magnetic field is
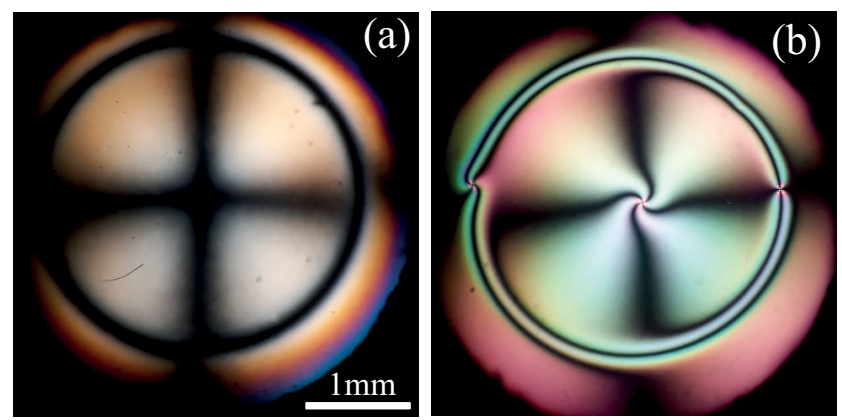

(c)

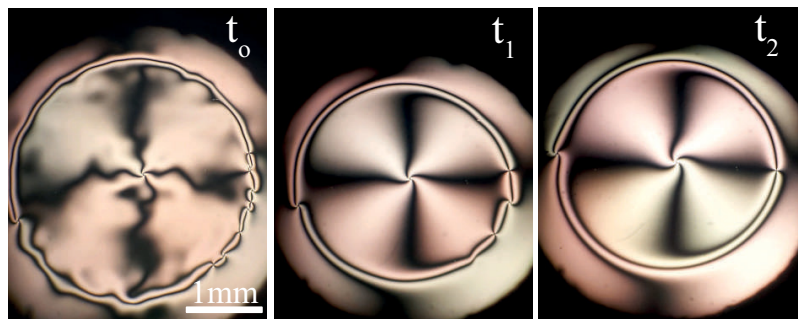

FIG. 2. Magnetic ring-induced vortex triplet in a nematic liquid crystal layer. (a) Snapshot of a vortex observed close to the Fréedericksz transition $V_{0}=6.3 \mathrm{~V}_{\mathrm{pp}}$. (b) Snapshot of the vortex triplet observed above the orientational transition $V_{0}=7.95 \mathrm{~V}_{\mathrm{pp}}$. (c) A temporal sequence of snapshots of the emergence of vortex triplet formation $t_{0}<t_{1}<t_{2}$, far from the orientational transition $V_{0}=$ $15.0 \mathrm{~V}_{\mathrm{pp}}$.

monitored by a complementary metal-oxide-semiconductor (CMOS) camera (Thorlabs DCC1645C), which allows us to observe the central zone of the magnetic ring.

Experimental observations of the vortex triplet. When the liquid crystal sample is only under the influence of the magnetic field, there is no light transmission. Hence, the torque generated by the magnetic field is not capable of overcoming the elastic resistance; namely, the considering magnetic ring is not capable of inducing the molecular reorientation. In order to generate this reorientation, a voltage drop is included orthogonal to the sample of the form $V_{\omega}(t)=V_{0} \cos (\omega t)$. Due to negative electrical anisotropy and the simultaneous presence of the electric and magnetic field, we observe the reorientation transition for voltages less than the Fréedericksz voltage (critical voltage for the reorientation instability purely induced by an electric field). By increasing the magnetic intensity by using magnets of different thicknesses, we observe a transition of molecular reorientation for lower voltages. Unexpectedly, close to the reorientation instability, the emergence of a vortex with a thick core in the center with a ring near to the inner radius of the magnet is observed. Figure 2 shows the typically observed vortices. By increasing the voltage, we have a better resolution to observe the emergent structure that is made up of a positive vortex in the center and two vortices on its lateral flanks of opposite charges, a vortex triplet [cf. Fig. 2(b)]. By turning off the voltage and then considering a higher voltage, the vortex structure emerges with a ring characterized by exhibiting multiple vortices with alternating topological charges. Subsequently, these vortices are annihilated by pairs, and only two survive in the antipodes (see the bottom panels in Fig. 2). The vortex triplet is the equilibrium state of the system. 


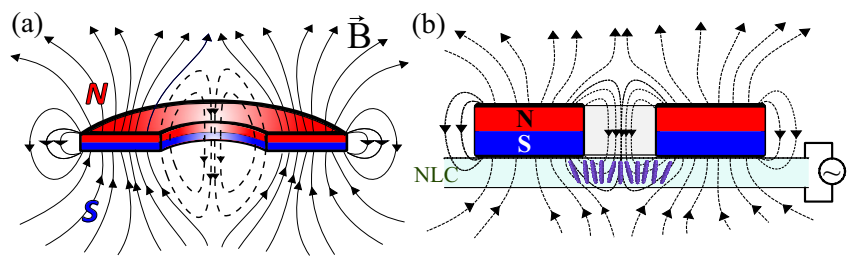

FIG. 3. Schematic representation of the magnetic field structure of a magnetic ring with a rectangular cross section. (a) Threedimensional representation of the magnetic field. The curves account for the lines of the magnetic field. Dashed lines account for the structure of the magnetic field within the magnetic protuberance. (b) Two-dimensional representation of the magnetic field of the ring onto the nematic liquid crystal layer. The rods stand for the average direction of molecules.

An intuitive explanation of the appearance of a vortex triplet. The induced vortex triplet is determined by the structure of the magnetic field generated by the magnetic ring. Since the magnet has a north and south pole parallel to the liquid crystal sample and a rectangular cross section, a magnetic protuberance is observed at the center of the ring [30]. Figure 3 schematically shows the magnetic structure of a ring with a rectangular cross section. Dashed curves account for the magnetic protuberance. This magnetic protuberance is a consequence of the boundary condition imposed by the ring's inner wall on the magnetic field. Liquid crystal molecules tend to orient orthogonally with the magnetic field as a consequence of the negative anisotropic magnetic susceptibility $[16,17]$. When considering the liquid crystal sample inside the magnetic protuberance, it naturally induces a positive vortex at the center and also a circular defect line that circumscribes the vortex [cf. Fig. 3(b)]. However, for homeotropic anchoring conditions, a defect line is unstable and always stabilizes in a set of vortices with alternating charges along the line $[16,17,31]$. Indeed, one expects this line to stabilize in vortices and, ultimately, only a few vortices to survive along the line. Observe that when the sample stands outside the magnetic protuberance, one only observes a single vortex, and there is no ring to circumscribe it [26]. Indeed, this can be accomplished by moving the liquid crystal sample away from the magnet.

Theoretical description of a vortex triplet. The structure of the magnetic protuberance can be modeled in a first approximation by the magnetic field of a cylindrical magnet of the form

$$
\vec{B}(\rho, z)=m\left[\frac{\left(3 z^{2}-\sigma\right) \hat{z}}{\left(\rho^{2}+z^{2}\right)^{5 / 2}}-\frac{l_{0}^{2} \hat{z}-3 z \rho \hat{\rho}}{\left(\rho^{2}+z^{2}\right)^{3 / 2}}\right]+b_{0} \hat{z},
$$

where $\sigma>0, l_{0}$, and $b_{0}$ are phenomenological dimensional parameters $\left(\mathrm{m}^{2}, \mathrm{~m}\right.$, and $\mathrm{T}$, respectively) that account for the geometric features of the magnet ring, $m$ is a constant that has a dimension of permeability per magnetic moment, and $\{z, \rho, \theta\}$ are the cylindrical coordinates. The origin of the coordinate is fixed at the center of the magnetic ring. Note that as a result of the azimuthal symmetry of the ring, the magnetic field does not depend on the $\theta$ coordinate.

To shed light on the mechanism of the creation and pinning of vortices, we derive a model in the vicinity of the reorientational transition. As nematic liquid crystal molecules are weakly tilted from the axis $\hat{z}$, the backflow effects can be neglected $[17,21,24]$. The molecular reorientation of the liquid crystal is described by the director vector $\vec{n}$ when the temperature is constant $[16,17]$. The dynamic of the director is characterized by minimizing the Frank-Ossen free energy

$$
\begin{aligned}
\mathcal{F}= & \int d V\left\{\frac{K_{1}}{2}(\nabla \cdot \vec{n})^{2}+\frac{K_{2}}{2}[\vec{n} \cdot(\nabla \times \vec{n})]^{2}\right. \\
& \left.+\frac{K_{3}}{2}[\vec{n} \times(\nabla \times \vec{n})]^{2}-\frac{\epsilon_{a}}{2}(\vec{E} \cdot \vec{n})^{2}-\frac{\chi_{a}}{2}(\vec{B} \cdot \vec{n})^{2}\right\},
\end{aligned}
$$

where $\left\{K_{1}, K_{2}, K_{3}\right\}$ are the liquid crystal elastic constants, and $\vec{E}$ and $\vec{B}$ are the electric and magnetic field, respectively $[16,17]$. We consider a uniform vertical electric field $\vec{E}=$ $E_{z} \hat{z}=V_{\omega}(t) / d \hat{z}$ and a magnetic field $\vec{B}=B_{z} \hat{z}+B_{\rho} \hat{\rho}$ given by formula (1).

To study the dynamics of the director, we will use the strategy, amplitude equation, presented in Ref. [24] and references therein. Close to the reorientational instability we use the ansatz

$$
\vec{n}=\left(\begin{array}{c}
\frac{\gamma \operatorname{Re}(A)}{a} \sin \left[\frac{\pi}{d}\left(z+\frac{h}{2}\right)\right] \\
\frac{\gamma \operatorname{Im}(A)}{a} \sin \left[\frac{\pi}{d}\left(z+\frac{h}{2}\right)\right] \\
1-\frac{\gamma^{2}|A|^{2}}{2 a^{2}} \sin ^{2}\left[\frac{\pi}{d}\left(z+\frac{h}{2}\right)\right]
\end{array}\right)+\text { HOT },
$$

where $A$ accounts for the amplitude of the critical mode, $a^{2} \equiv\left[\left(K_{1}-K_{3}\right)(\pi / d)^{2}-3 \epsilon_{a} E_{z}^{2}-3 \chi_{a} B_{z}^{2}\right] / 4$ is a normalization constant, and HOT stands for high-order terms in the critical amplitude. Using the above ansatz, Eq. (3), on the Frank free energy (2) and minimizing after straightforward calculations, one gets the amplitude equation (the dimensionless topologically driven Ginzburg-Landau equation)

$$
\gamma_{0} \partial_{t} A=\mu(\rho) A-|A|^{2} A+\nabla^{2} A+\delta \partial_{\eta \eta} \bar{A}+f(\rho) e^{i \theta},
$$

where the spatial coordinates have been scaled by $\vec{r} \rightarrow$ $\vec{r} \sqrt{2 /\left(K_{1}+K_{2}\right)}, \mu(\rho)=\mu_{0}+\mu_{1}(\rho)$ is the inhomogeneous bifurcation parameter, $\mu_{0} \equiv-K_{3}(\pi / d)^{2}-\epsilon_{a} E_{z}^{2}$ is the bifurcation parameter related to the electrical Fréedericksz transition, $\mu_{1}(\rho) \equiv \chi_{a} B_{z}^{2}\left(\rho, z_{0}\right)$ is the inhomogeneous modification of the reorientational transition, $z_{0}$ is the height of the liquid crystal sample, $\delta=\left(K_{1}-K_{2}\right) /\left(K_{1}+K_{2}\right)$ accounts for the elastic anisotropy of the liquid crystal, and $\partial_{\eta} \equiv \partial_{x}+i \partial_{y}$ is a differential operator in the complex plane; note that the Laplacian satisfies $\nabla^{2}=\partial_{\eta, \bar{\eta}}$, and $f(\rho)=$ $4 \chi_{a} a^{1 / 4} B_{\rho}\left(\rho, z_{0}\right) B_{z}\left(\rho, z_{0}\right) /(\gamma \pi)$ accounts for the strength of the topological forcing. Notice a similar equation has been derived for liquid crystals with photosensitive walls but with a Gaussian forcing [21,24], in which vortex induction has been demonstrated through the light-matter interaction. Figure 4 shows in the left panels the bifurcation parameter and the forcing as a function of the radial coordinate. Notice that the vortices are placed at the zeros of the forcing. $f(\rho)$ is annulled at the origin and in the circumference of radius $\rho^{*}$. In the regime below the electrical Fréedericksz transition, $\mu_{0} \lesssim 0$, the system exhibits an analytical approximation of the form $A \approx f(r) e^{i \theta} / \mu_{0}$ [32]. Indeed, this solution is characterized by exhibiting a vortex at the center and also a circular defect line that circumscribes the vortex. Figure 4(c) shows numerical simulations of Eq. (4) for $\mu_{0}<0$. From the polarized field $\psi(x, y) \equiv \operatorname{Re}(A) \operatorname{Im}(A)$ (top panel) [24], we only 

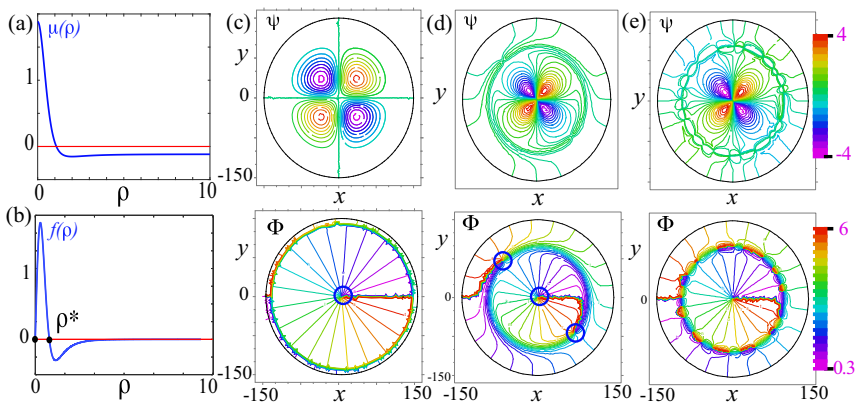

FIG. 4. Numerical simulation of a vortex triplex obtained from the topologically driven Ginzburg-Landau Eq. (4) with $z_{0}=28$, $\sigma=28, m=13000, l_{0}=1, b_{0}=0, \gamma_{0}=1$, and $\delta=0.1$. Profile of the inhomogeneous bifurcation parameter $\mu(\rho)$ (a) and topological forcing strength $f(\rho)$ (b) as a function of the radial coordinate. Contour plot of the polarized field $\psi(x, y)=\operatorname{Re}(A) \operatorname{Im}(A)$ (top panel) and phase field $\Phi(x, y)=\arctan [\operatorname{Im}(A) / \operatorname{Re}(A)]$ (bottom panel) of the complex amplitude $A$, below $\mu_{0}<0\left(\mu_{0}=-1\right)$ (c) and above $\mu_{0}>0\left[\mu_{0}=0.2\right.$ (d) and $\mu_{0}=3.0$ (e) $]$ the Fréedericksz voltage. Inserted circles highlight phase singularities.

detect the central vortex with a cross shape. The ring that circumscribes the central vortex is not detected because it has a small amplitude. This ring is only detected in the phase field $\Phi(x, y)=\arctan [\operatorname{Im}(A) / \operatorname{Re}(A)]$ (bottom panel), and it has two singularities of opposite charges in its antipodes. Numerical simulations of model Eq. (4) were conducted considering a triangular finite-element code with adaptive spatial and temporal steps, and a simulation box of dimensions $300 \times 300$ with a Neumann boundary condition was considered. Increasing the bifurcation parameter, the central vortex swirls, and the ring that encloses the amplitude notoriously shows two vortices of opposite charges at the antipodes [see Fig. 4(d)], which is what we observe in experiments (cf. Fig. 2). Starting from $A=0$ and considering a larger bifurcation parameter, we see the vortex emerge at the center with a circular defect line that destabilizes into a set of vortices that begin to interact and annihilate in pairs [see Fig. 4(e)]. Note that a similar phenomenon is observed in the experiment [cf. Fig. 2(c)]. Hence, model Eq. (4) describes the emergence of a vortex triplet induced by the combined action of a magnetic ring and electric field into a nematic liquid crystal layer (see Figs. 2 and 4).

Unexpectedly, when we decrease the frequency to a fraction of $\mathrm{Hz}$, experimentally, the vortex triplet is destabilized from both the central vortex and the ring that circumscribes it by the induction of vortices, resulting in a front of vortices invading the system, as it is illustrated in Fig. 5 (see Videos 1 and 2 in the Supplemental Material [33], which are in real time). The vortex lattice corresponds to the equilibrium state of the system. Notice that the vortex lattice is characterized by vibrating at the frequency of the electrical forcing. The emergence of the vortex lattice is due to the combined effect of the oscillatory and magnetic electric field. Indeed, the magnetic and electric field or thermal gradient can couple with the fluid and generate charge movements [34-38], a Carr-Helfrich type mechanism [17], that affect the stability of the triplet. When the low-frequency oscillatory behavior of the electric field $E_{z}(t)$ is included, one cannot neglect the inertia in the

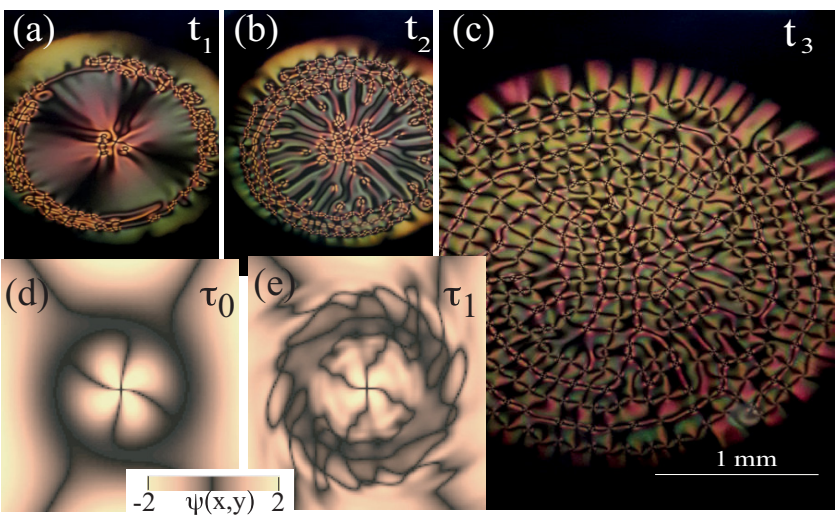

FIG. 5. Vortex lattices. (a)-(c) A temporal sequence of snapshots of the emergence of a vortex lattice from a vortex triplet $t_{1}<t_{1}<$ $t_{3}$, with $V_{0}=9.0 \mathrm{~V}_{\mathrm{pp}}$ and $\omega=10 \mathrm{mHz}$. (d), (e) Numerical temporal sequence of a vortex lattice, $\tau_{0}<\tau_{1}$, obtained from the amplitude Eq. (5) with $z_{0}=15, \sigma=2000, m=1000, l_{0}=1, b_{0}=0.55, \gamma_{0}=$ $0.5, \delta=0.8, \Gamma=1$, and $\omega=1$.

dynamics of the director. Then the amplitude equation takes the form

$$
\partial_{t t} A+\gamma_{0} \partial_{t} A=\mu A-|A|^{2} A+\nabla^{2} A+\delta \partial_{\eta \eta} \bar{A}+f(\rho) e^{i \theta},
$$

where $\mu=\mu_{0}+\mu_{1}(\rho)+\Gamma \sin (2 \omega t)$. For large $\gamma_{0}$ and high frequencies, this model equation is approximated by Eq. (4). Namely, this model presents a triplet vortex solution. However, when the frequency is decreased, the amplitude $A$ begins to oscillate. For a critical frequency, the ring around the vortex becomes unstable. This instability generates the emergence of vortex pairs. Figure 5 shows the typically observed vortex lattice structure and the triplet that becomes unstable (see Video 3 in the Supplemental Material [33]).

In summary, the combined effect of the magnetic field of a magnetic ring and the uniform electric field onto a homeotropic nematic liquid crystal cell induces a stable vortex triplet and vortex lattice. An amplitude equation allows us to reveal the origin of the vortex triplet and the emergence of the vortex lattice. Theoretical findings show a quite fair agreement with experimental observations. The generated vortex lattices are obtained in a simple configuration that does not require a complex structure of electrodes, inhomogeneities, applications of thermal gradients, or photosensitive walls. The characterization and properties of vortex lattices are in progress.

The observation of vortex lattices as a consequence of oscillatory fields corresponds to a topological transition out of equilibrium. Hence, the presence of a constant electric or magnetic field is not relevant for the observation of a topological lattice [35-38]. It is expected that this type of phenomenon can be generic in systems that present vortices. Our finding enables another avenue of topology transitions in systems driven by periodic forcing.

The authors thank M. Kowalczyk for fruitful discussions. This work was funded by ANID-Millennium Science Initiative Program-ICN17_012. M.G.C. is thankful for financial support from the Fondecyt 1180903 project. 
[1] A. L. Fetter and J. D. Walecka, Theoretical Mechanics of Particles and Continua (Courier Corporation, North Chelmsford, MA, 2003).

[2] G. Nicolis and I. Prigogine, Self-Organization in Nonequilibrium Systems (Wiley, New York, 1977).

[3] L. M. Pismen, Patterns and Interfaces in Dissipative Dynamics (Springer, Berlin, 2006).

[4] M. Cross and H. Greenside, Pattern Formation and Dynamics in Nonequilibrium Systems (Cambridge University Press, New York, 2009).

[5] A. C. Newell, Solitons in Mathematics and Physics (Society for Industrial and Applied Mathematics, Philadelphia, 1985).

[6] M. Remoissenet, Waves Called Solitons: Concepts and Experiments (Springer, Heidelberg, 1993).

[7] Dissipative Solitons: From Optics to Biology and Medicine, edited by N. Akhmediev and A. Ankiewicz, Lecture Notes in Physics (Springer, Heidelberg, 2008), Vol. 751.

[8] H. G. Purwins, H. U. Bodeker, and S. Amiranashvili, Dissipative solitons, Adv. Phys. 59, 485 (2010).

[9] O. Descalzi, M. G. Clerc, S. Residori, and G. Assanto, Localized States in Physics: Solitons and Patterns (Springer, Berlin, 2011).

[10] M. Tlidi, K. Staliunas, K. Panajotov, A. G. Vladimiorv, and M. Clerc, Localized structures in dissipative media: From optics to plant ecology, Philos. Trans. R. Soc., A 372, 20140101 (2014).

[11] P. Coullet, Localized patterns and fronts in nonequilibrium systems, Int. J. Bifurcation Chaos Appl. Sci. Eng. 12, 2445 (2002).

[12] L. M. Pismen, Vortices in Nonlinear Fields (Oxford Science, New York, 1999).

[13] I. S. Aranson and L. Kramer, The world of the complex Ginzburg-Landau equation, Rev. Mod. Phys. 74, 99 (2002).

[14] E. Sandier and S. Serfaty, Vortices in the Magnetic GinzburgLandau Model (Springer, Boston, 2008).

[15] F. Bethuel, H. Brezis, and F. Helein, Ginzburg-Landau Vortices (Springer, New York, 2012).

[16] S. Chandrasekhar, Liquid Crystals (Cambridge University Press, Cambridge, UK, 1992).

[17] P. G. de Gennes and J. Prost, The Physics of Liquid Crystals, 2nd ed. (Oxford Science/Clarendon, Oxford, UK, 1993).

[18] V. Freedericksz and V. Zolina, Forces causing the orientation of an anisotropic liquid, Trans. Faraday Soc. 29, 919 (1927).

[19] E. Brasselet, N. Murazawa, H. Misawa, and S. Juodkazis, Optical Vortices from Liquid Crystal Droplets, Phys. Rev. Lett. 103, 103903 (2009).

[20] E. Brasselet and C. Loussert, Electrically controlled topological defects in liquid crystals as tunable spin-orbit encoders for photons, Opt. Lett. 36, 719 (2011).

[21] R. Barboza, U. Bortolozzo, G. Assanto, E. Vidal-Henriquez, M. G. Clerc, and S. Residori, Vortex Induction via Anisotropy Stabilized Light-Matter Interaction, Phys. Rev. Lett. 109, 143901 (2012).

[22] E. Brasselet, Tunable Optical Vortex Arrays from a Single Nematic Topological Defect, Phys. Rev. Lett. 108, 087801 (2012).
[23] M. El Ketara and E. Brasselet, Self-induced nonlinear spin-orbit interaction of light in liquid crystals, Opt. Lett. 37, 602 (2012).

[24] R. Barboza, U. Bortolozzo, M. G. Clerc, S. Residori, and E. Vidal-Henriquez, Optical vortex induction via light-matter interaction in liquid-crystal medial, Adv. Opt. Photonics 7, 635 (2015).

[25] P. Pieranski, B. Yang, L.-J. Burtz, A. Camu, and F. Simonetti, Generation of umbilics by magnets and flows, Liq. Cryst. 40 , 1593 (2013).

[26] E. Brasselet, Tunable High-Resolution Macroscopic SelfEngineered Geometric Phase Optical Elements, Phys. Rev. Lett. 121, 033901 (2018).

[27] H. H. Arnaut and G. A. Barbosa, Orbital and Intrinsic Angular Momentum of Single Photons and Entangled Pairs of Photons Generated by Parametric Down-Conversion, Phys. Rev. Lett. 85, 286 (2000).

[28] F. Tamburini, G. Anzolin, G. Umbriaco, A. Bianchini, and C. Barbieri, Overcoming the Rayleigh Criterion Limit with Optical Vortices, Phys. Rev. Lett. 97, 163903 (2006).

[29] J. Wang, J.-Y. Yang, I. M. Fazal, N. Ahmed, Y. Yan, H. Huang, Y. Ren, Y. Yue, S. Dolinar, M. Tur, and A. E. Willner, Terabit free-space data transmission employing orbital angular momentum multiplexing, Nat. Photonics 6, 488 (2012).

[30] I. Lebedev, Tekh. Molodezhi 6, 2 (1991) (see also http://nt.ru/tp/mr/mmk.htm); M. F. Ostrikov, New Manifestations of Magnetism (St. Petersburg, 1994).

[31] J. M. Gilli, M. Morabito, and T. Frisch, Ising-Bloch transition in a nematic liquid crystal, J. Phys. II (France) 4, 319 (1994).

[32] E. Calisto, M. G. Clerc, M. Kowalczyk, and P. Smyrnelis, On the origin of the optical vortex lattices in an nematic liquid crystal light valve, Opt. Lett. 44, 2947 (2019).

[33] See Supplemental Material at http://link.aps.org/supplemental/ 10.1103/PhysRevResearch.2.042026 for movies that show the destabilization of the central vortex and the surrounding ring in real time (Video 1), the optical vortex lattice induced by the magnetic ring and oscillatory electric field in real time (Video 2 ), and the vortex structures that emerge numerically for the model Eq. (5) (Video 3).

[34] P. Pieranski, E. Dubois-Violette, and E. Guyon, Heat Convection in Liquid Crystals Heated from Above, Phys. Rev. Lett. 30, 736 (1973).

[35] Y. Sasaki, V. S. R. Jampani, C. Tanaka, N. Sakurai, S. Sakane, K. V. Le, F. Araoka, and H. Orihara, Large-scale selforganization of reconfigurable topological defect networks in nematic liquid crystals, Nat. Commun. 7, 1 (2016).

[36] M. Kim and F. Serra, Tunable large-scale regular array of topological defects in nematic liquid crystals, RSC Adv. 8, 35640 (2018).

[37] L. K. Migara and J. K. Song, Standing wave-mediated molecular reorientation and spontaneous formation of tunable, concentric defect arrays in liquid crystal cells, NPG Asia Mater. 10, e459 (2018).

[38] M. G. Clerc, M. Kowalczyk, and V. Zambra, Exotic states of matter in an oscillatory driven liquid crystal cell, arXiv:2009.06528. 